\title{
Notes of the editorial board on the role of medical physics in radiotherapy
}

\author{
Waldemar Ulmer \\ Klinikum München-Pasing, Department of Radiation Therapy and MPI of Physics, Göttingen, Germany
}

Received September 7, 2013; Accepted September 8, 2013; Published Online September 09, 2013

\section{Editorial}

The radiotherapy of malignant diseases has reached much progress during the past decade. Thus, intensity modulated radiation therapy (IMRT), RapidArc and Stereotaxy now belong to the standard modalities of tumor treatment with high energy radiation in clinical practice. In recent time, the particle therapy with protons and partially with heavy carbon ions has reached an important completion of these modalities with regard to some suitable applications. In spite of this enrichment essential features need further research activities and publications in this field: Nuclear reactions and the role of the released neutrons; electron capture of positively charged nuclei at lower projectile energies (e.g. in the environment of the Bragg peak and at the distal end of the particle track); correct dose delivery in scanning methods by accounting for the influence of the lateral scatter of beamlets. ${ }^{1-7}$ Deconvolution methods can help to overcome these problems ${ }^{4}$, which already occur in radiotherapy of very small photon beams. ${ }^{8}$

With regard to studies of clinical/radiobiological implications the accurate knowledge of the described problems is an essential feature and starting point. We particular mention the influence of released neutrons and the electron capture to the LET and RBE. In particular, electron capture of heavy ions such as carbon influences many aspects of this therapy modality and the superiority of these ions is drastically reduced in the domain of the Bragg peak. ${ }^{3}$ Figures $1-3$ make apparent the influence of electron capture for heavy carbon ions. Thus, all positively charged projectile show in the high energy domain a stripping effect, i.e. the charged projectile

Corresponding author: Waldemar Ulmer, PhD; Klinikum München-Pasing, Department of Radiation Therapy and MPI of Physics, Göttingen, Germany;

Email: Waldemar.Ulmer@gmx.net

Cite this article as:

Ulmer W. Notes of the editorial board on the role of medical physics in radiotherapy. Int J Cancer Ther Oncol 2013;1(1):01014.

DOI: $10.14319 /$ ijcto.0101.4

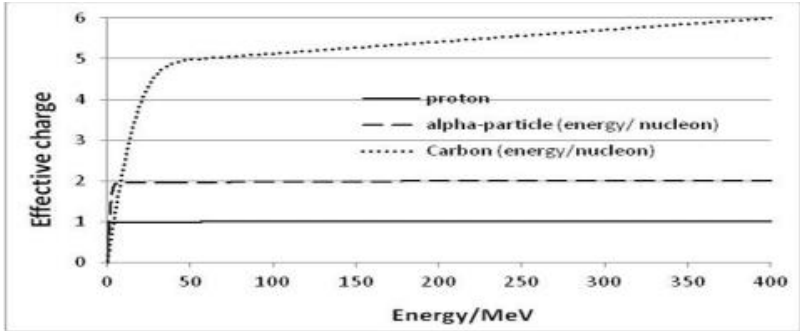

FIG. 1: Effective charge of the projectile nuclei proton, $\alpha$-particle and carbon. The initial energy amounts to $400 \mathrm{MeV} /$ nucleon.

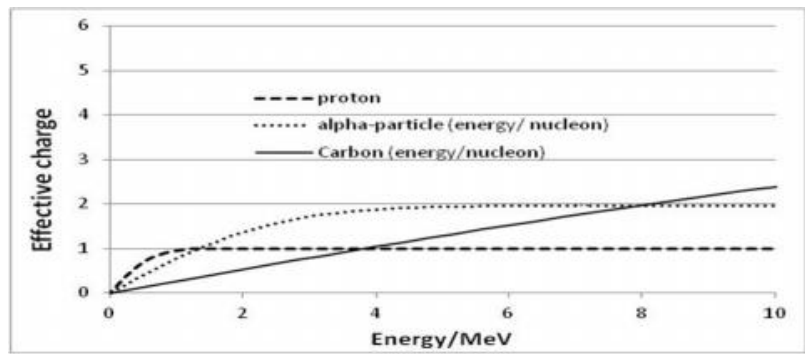

FIG. 2: Effective charge of the three projectile nuclei in the low energy region of Figure 1.

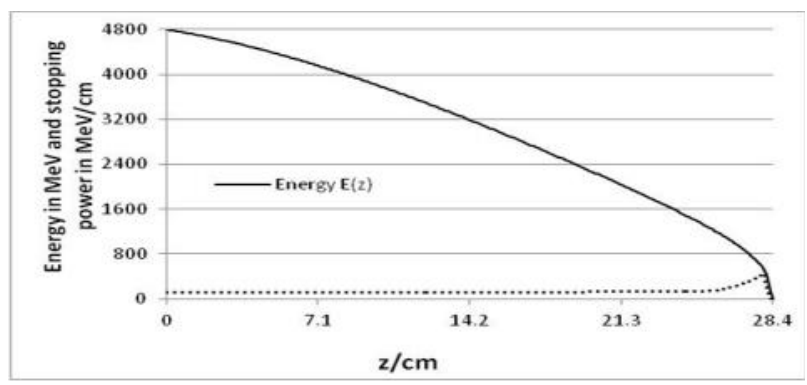

FIG. 3: $\mathrm{E}(\mathrm{z})$ versus $\mathrm{dE} / \mathrm{dz}$ of a carbon ion in dependence of the depth $\mathrm{z}$; the initial energy amounts to $400 \mathrm{MeV} /$ nucleon.

(With regard to the Figures $1-3$ it should be mentioned that independent of the initial energy (e.g 500 MeV/nucleon or 300 MeV/nucleon) the initial charge of the impinging carbon ion is always $q=6+$. The publication ${ }^{3}$ also presents effective charges $q(E)$ of carbon ions with different initial energy.)

captures a surrounding electron, but this electron goes lost before a transition to a free shell can occur (Barkas effect). Only at lower projectile velocities the transition can occur. 
In the case of carbon ions we have to consider a cascade of transitions to free atomic shells of carbon. At first, $\left(\mathrm{C}_{6+}\right)^{12}$ will be changed to $\left(\mathrm{C}_{6,5+}\right)^{12}$, and this electron capture effect is repeated, until the carbon ion is transformed to $\left(\mathrm{C}_{6,1+}\right)^{12}$ in the Bragg peak domain. At the distal end we obtain neutral carbon (or helium or hydrogen). Thus in the Bragg peak region the difference between the three projectile nuclei is rather small. Compared to protons the significantly increased importance of nuclear reactions and related fission products of carbon ions represents a further rather unresolved problem in the case of heterogeneous media. Contributions to these aspects can be found in the publications below, which should stimulate other authors to consider further research projects in this field.

\section{References}

1. Ulmer W, Matsinos E. Theoretical methods for the calculation of Bragg curves and 3D distribution of proton beams. Eur Phys J Special Topics 2010; 190: $1-81$.

2. Ulmer W, Schaffner B. Foundation of an analytical proton beamlet model for inclusion in a general proton dose calculation system. Radiation Physics and Chemistry 2011; 80: 378-402.

3. Ulmer $\mathrm{W}$. The role of electron capture and energy exchange of positively charged particles passing through matter. Journal of Nuclear and Particle Physics 2012; 2: 77-86.

4. Ulmer W. Deconvolution of a linear combination of Gaussian kernels by Liouville-Neumann series applied to an integral equation of second kind with applications to radiation physics/image processing. An Introductory Guide to Digital Image Processing (Edited by: A. Mishra). iConcept Press ISBN:

978-14775548-0-7 (2012).
5. Paganetti H. Nuclear interactions in proton therapy: dose and relative biological effect distributions originating from primary and secondary particles. Phys Med Biol 2002; 47: 747-62.

6. Gottschalk B, Platais R,Platais H. Nuclear interactions of $160 \mathrm{MeV}$ protons stopping in copper: A test of Monte Carlo nuclear models. MedPhys1999; 26:2597 - 2601.

7. Zhang R, Newhauser W. Calculation of water equivalent thickness of materials of arbitrary density, elemental composition and thickness in proton beam irradiation. Phys Med Biol 2009; 54: 1383-95.

8. Fan Y, Nath R. Intensity modulation under geometrical uncertainty: a deconvolution approach to robust fluence. Phys Med Biol 2010; 55:4029-45.

(Dr. W. Ulmer is an editorial board member of International Journal of Cancer Therapy and Oncology) 